\title{
Current Technical Issues for Surgery of Primary Liver Cancer
}

\author{
Yoshikuni Kawaguchia Goro Honda ${ }^{b}$ Itaru Endo ${ }^{c}$ \\ Daniel Cherquid Norihiro Kokudoa
}

aHepato-Biliary-Pancreatic Surgery Division, Department of Surgery, Graduate School of Medicine, University of Tokyo, Tokyo, b Department of Surgery, Tokyo Metropolitan Cancer and Infectious Diseases Center Komagome Hospital, Tokyo, 'Department of Gastroenterological Surgery, Graduate School of Medicine, Yokohama City University, Yokohama, Japan, dHepatobiliary Center, Paul Brousse Hospital, Université Paris Sud, Paris, France

\section{Key Words}

Fluorescence imaging technique $\cdot$ Hepatocellular carcinoma .

Intrahepatic cholangiocellular carcinoma - Laparoscopic liver resection .

Three-dimensional computed tomography

\begin{abstract}
Primary liver cancer is the fifth most common cancer worldwide. Apart from liver transplantation, surgical resection has been accepted as the effective local treatment for hepatocellular carcinoma (HCC), one of the most common primary liver cancers. Recent technological innovations including navigation technology and intraoperative real-time fluorescence guidance have been utilized for liver resections in clinical practice. With respect to liver resection techniques, the laparoscopic approach has been increasingly gaining popularity as one of the minimally-invasive treatments of HCC. These technological innovations and technical advancements are expected to further improve the safety and efficacy of liver resections.
\end{abstract}

Copyright $\odot 2016$ S. Karger AG, Basel

\section{Introduction}

Primary liver cancer is the fifth most common cancer worldwide and the second leading cause of cancer-related death among men, whereas, for women, it is the seventh most com- 
mon cancer and the sixth leading cause of cancer-related death [1], respectively. Apart from liver transplantation, surgical resection has been accepted as an effective local treatment for hepatocellular carcinoma (HCC). Recent technological innovations and advances in surgical techniques have improved the safety of liver resections [2]. Moreover, the laparoscopic approach has been increasingly gaining popularity as one of the minimally-invasive treatments of HCC [3].

This article focuses on technical issues for the surgery of HCC, including navigation technology, fluorescence-guided liver surgery, and laparoscopic liver resection (LLR). We aim to update the reader with respect to the current management of HCC from the surgical point of view in clinical practice.

\section{Preoperative and Intraoperative Navigation Technology}

Several therapeutic modalities such as major vessel resection, portal vein embolization and perioperative chemotherapy have led to better long-term survival for intrahepatic cholangiocarcinoma [4]. Although the safety of hepatic resection has improved, there are still many cases in which patients have developed serious postoperative complications. Kenjo et al. analyzed a national clinical database of over 7,000 patients who underwent hepatectomy [5]. The 90-day in-hospital mortality was $4.0 \%$. It was significantly higher in the cohort who received right hemihepatectomy with caudate lobe resection $(9.5 \%)$ and left trisectionectomy with caudate lobe resection (14.6\%), respectively. There are several reasons for severe postoperative complications such as the deterioration of the patients' general conditions, inappropriate preoperative evaluation of functional reserve of the liver remnant, and intraoperative injuries to the vasculature due to atypical anatomy. The latter problem can be avoided if surgeons are aware of such anatomical variations before surgery.

There are several reports presenting the usefulness of preoperative three-dimension (3D) simulation [6, 7]. For example, it is difficult to realize the branching patterns of the segmental hepatic artery through two-dimension computed tomography (CT) alone. A variation of the right posterior segmental artery sometimes becomes an issue in the surgery for perihilar tumors [8]. Virtual 3D-CT imaging clearly shows this kind of variation (fig. 1a and b). In this case, we planned left trisectionectomy with resection of the portal vein and right hepatic artery. The posterior hepatic artery was not visible at the start of the operation since it ran behind the right anterior portal vein. Therefore, 3D-CT simulation is indispensable for avoiding injury to the arteries in such instances.

Variations of the middle hepatic vein (MHV) tributaries can sometimes be another issue. Image-based computer assistance preoperatively enables the identification of areas at risk of devascularization or venous congestion, in addition to the volumetric analysis of such areas [6]. For example, a dominant MHV may occasionally run within the anterior segment (fig. 1c and d). The venous flow of the anterior segment and even a part of posterior segment drains into the MHV. Reconstruction of the MHV may be necessary for tumors in the left liver, which are adjacent to or involving such dominant MHV. This is especially relevant when a large congestive area appears on the surface of the anterior segment by a test-clamp of the MHV in the small liver remnant. If the patient has venous collaterals between the middle and right hepatic vein, reconstruction of the MHV may be unnecessary. However, such information can only become available during surgery through hepatic vein clamping. Operative planning for each patient based on the analysis of their own anatomy using virtual 3D images is expected to reduce postoperative complications. 


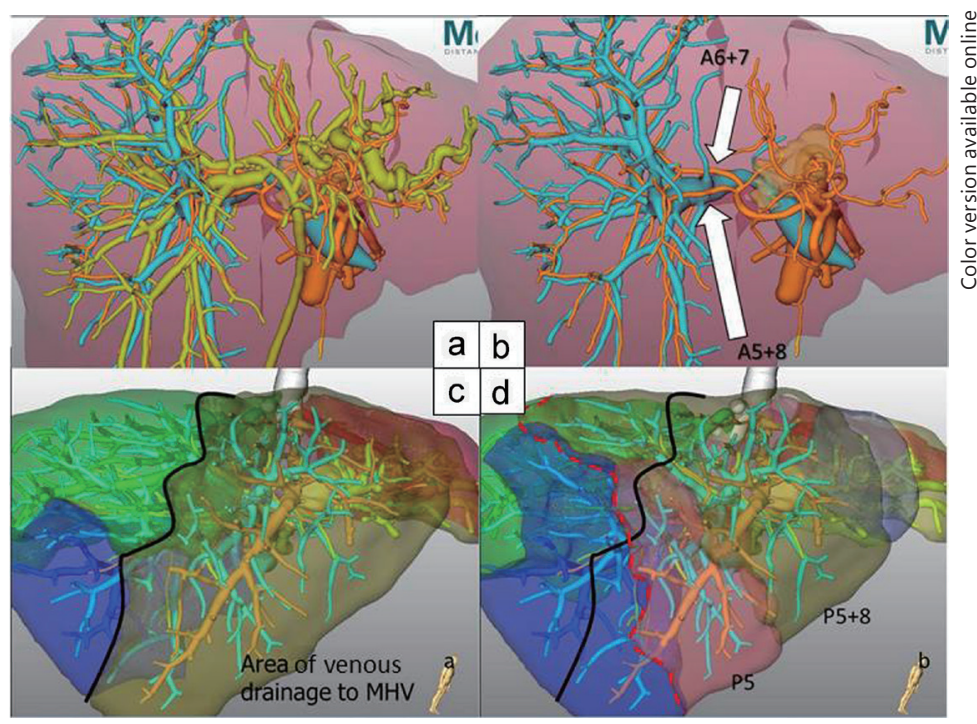

Fig. 1. Variations of the right hepatic artery ( $\mathbf{a}$ and $\mathbf{b}$ ). Drainage area of the MHV is visualized (c). Part of posterior segment is drained to the MHV (d).

\section{Fluorescence Image-Guided Liver Surgery}

Fluorescence imaging using indocyanine green (ICG) as a fluorescence source has been clinically applied for the visualization of bile and liver cancers in hepatobiliary surgery $[9,10]$. This technique can be utilized for visualizing hepatic regions of interest during liver resection. Classically, the regions supplied by the portal vein are identified with the puncture of the corresponding portal branches with subsequent dye injection under the guidance of intraoperative ultrasonography [11]. The regions drained by the hepatic veins are visualized through temporal arterial clamping [12]. This method facilitates anatomic liver resection and the identification of the extent of veno-occlusive regions. However, they do not always clearly visualize the regions of interest especially when the liver surface is covered with thick adhesive tissues and/or when the liver presents with severe cirrhosis. We herein detail ICG-fluorescence imaging for the visualization of regions supplied by the portal vein and drained by the hepatic veins.

\section{Identification of Regions Supplied by the Portal Vein}

A commercially available fluorescence imaging system (PDE-neo ${ }^{\circledR}$, Hamamatsu Photonics) was used. The camera head of the system was positioned $20 \mathrm{~cm}$ above the liver surface. ICG (0.25 mg=0.1 ml ICG; Diagnogreen ${ }^{\circledR}$, Daiichi Sankyo, Tokyo, Japan) was diluted in $5 \mathrm{ml}$ of indigo-carmine solution (20 mg, Daiichi Sankyo) [13]. The portal vein feeding the cancerbearing hepatic segment 8 was visualized longitudinally and punctured with a 22 gauge needle under intraoperative ultrasonographic guidance. The ICG with indigo-carmine was subsequently injected. Indigo-carmine staining was unclear due to the cirrhotic change of the liver surface (fig. 2a). However, ICG-fluorescence imaging was clearly visualized in hepatic segment 8 . Subsequently, the anatomic segment 8 resection was performed.

\section{Identification of Regions Drained by the Hepatic Vein}

Extended left hepatectomy with excision of the MHV was performed (fig. 2b), and the stump of the MHV was reconstructed using a cryopreserved vein graft (fig. 2c). After intrave- 
a

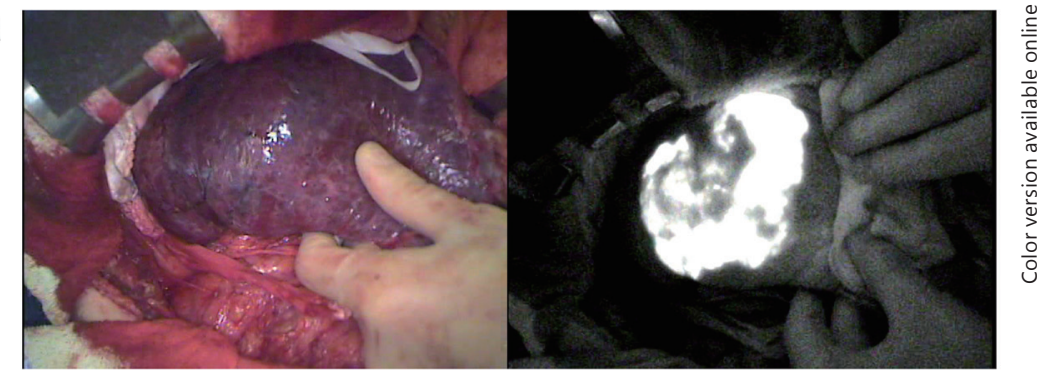

b
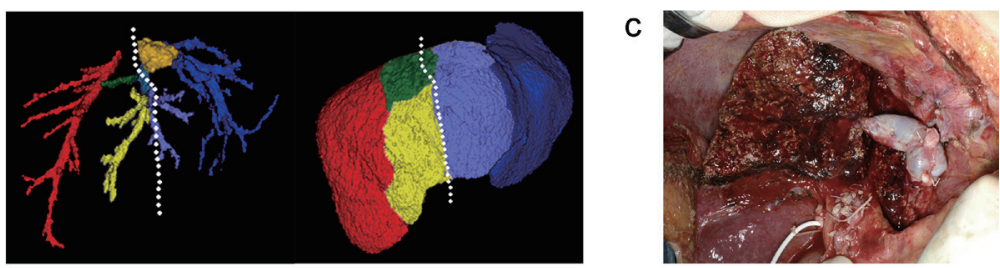

d

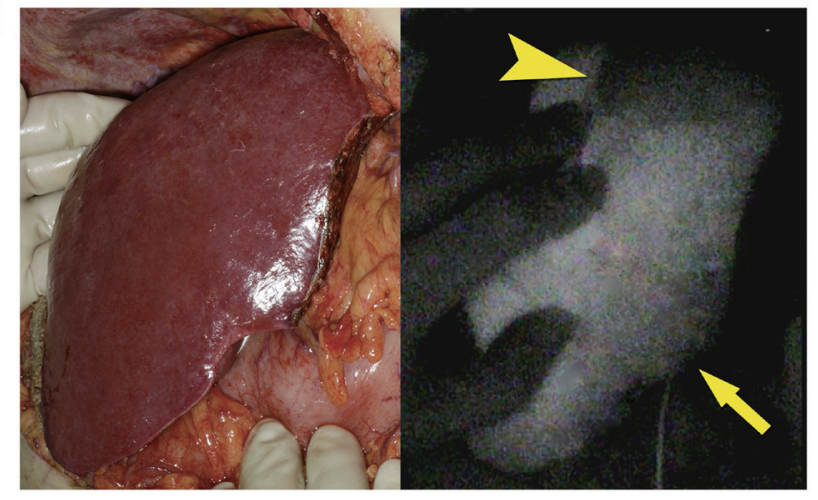

Fig. 2. Fluorescence imaging. a Gross appearance of the liver surface after dye injection (left) and corresponding fluorescence image (right). b Tumor location and planned resection line (white dotted line) were demonstrated on preoperative 3DCT. The remnant liver volume was estimated to be $429 \mathrm{ml}$, consisting of non-veno-occlusive regions drained by the right hepatic vein $(246 \mathrm{ml})$ and of veno-occlusive regions resulting from the division of the MHV tributaries draining segment 5 (V5) (163 ml) and segment 8 (V8) $(30 \mathrm{ml})$. $\mathbf{c}$ Intraoperative gross appearance following extended left hepatectomy with excision and reconstruction of the MHV tributaries. d Fluorescence imaging following intravenous injection of ICG reveals the demarcation between the region drained by the reconstructed vein (arrow) and that with venous occlusion (arrowhead) (From left to right, gross appearance and fluorescence image 240 seconds after the injection).

nous injection of ICG ( $2.5 \mu \mathrm{g} / \mathrm{ml}$ of the remnant liver volume), fluorescence imaging visualized the heterogeneous fluorescence intensity (FI) in the anterior sector. FI was lower in the cranial part than in the caudal part (fig. 2d), respectively. This implies that the venous outflow of cranial part was impaired due to the lack of venous reconstruction $[14,15]$. The venous outflow was determined to be preserved in a large part of the anterior sector, and further reconstruction was therefore not undertaken.

\section{Summary}

The classical methods for visualization of the portal and venous regions are useful and applicable without an additional imaging system. However, ICG-fluorescence imaging can clearly visualize an ill-defined margin of the portal segment under the conventional method and identify the regions with impaired venous outflow. The technique facilitates anatomic 
liver resection and improves the safety of liver resections that may cause venous occlusion in the remnant liver.

\section{LLR}

\section{Western Perspectives}

Despite a slower development than in other areas of abdominal surgery, LLR is rapidly increasing in popularity [16]. LLR is a difficult procedure requiring the expertise in both liver surgery and advanced laparoscopy. Mastering only simple laparoscopic procedures, such as cholecystectomy, is clearly insufficient and a whole new set of skills have to be learned by the "open" liver surgeon, including complex dissection and suturing techniques. The development of highly efficient new technologies has greatly enhanced those possibilities, including enhanced video equipment, electrosurgical devices, and staplers. The recent consensus conference held in Morioka, Japan acknowledged that minor resections are now considered standard practice while major resections and complex anatomical resections (segmentectomy 7 or 8, and sectionectomies) are still at an evaluation stage [3]. During the conference, a difficulty scoring system was also proposed taking into account the size and location of lesions, the extent of resection and the quality of the underlying liver disease (Iwate Criteria).

Interestingly, HCC occurring mainly in patients with cirrhosis, is the most common single reported indication for LLR [3]. This is the result of screening programs permitting a frequent diagnosis of early single HCCs. Earlier, our group observed that LLR was very well tolerated by patients with cirrhosis [17]. In particular, we observed much less postoperative ascites than previously experienced after open surgery. This was confirmed in several retrospective comparative studies and meta-analyses that suggested additional advantages such as decreased pain, bleeding, morbidity and hospital stay using the laparoscopic approach as compared with open surgery $[18,19]$. Surgical margins and long-term survival were found not to be inferior [20]. The recognition of the efficacy and safety of LLR for HCC has led to the increased number of cases performed, especially among patients with peripheral tumors less than $5 \mathrm{~cm}[21,22]$.

In Western countries where cadaveric donors organs are available, patients with early HCC are considered candidates for liver transplantation [23], a definitive treatment eradicating both the tumor and the underlying liver disease. This is obviously the case in the presence of associated decompensated cirrhosis, but it is also largely considered for patients with preserved liver function. The question of resection versus transplantation is more significant than in Eastern countries where living donors are mostly available and where cadaveric organ availability is sparse. In view that liver transplantation is limited, there has been regained interest for liver resection. While resection offers excellent overall survival in selected patients, it is hampered with high tumor recurrence rates. Close patient follow-up allows treatment of post-resection recurrences, including repeat resection and salvage liver transplantation [21, 24-26], with the advantages of initial LLR that clearly facilitates repeat surgery [27].

In conclusion, there are data suggesting that laparoscopic surgery can enhance the possibilities of resection in patients with early HCC and, for cases with favorable tumor locations, LLR should clearly be preferred. The place of resection compared to other curative treatments in patients who may be curable must be discussed on an individual basis in a multidisciplinary setting. These strategies may appropriately result in a more effective utilization of the limited supply of donor organs. 


\section{Eastern Perspectives}

Since anatomical hepatectomy $(\mathrm{AH})$ was proposed by Makuuchi et al., it has developed as an ideal procedure for the treatment of HCC in Japan [11]. Also for laparoscopic hepatectomy, which has recently been disseminated worldwide, $\mathrm{AH}$ is required for the treatment of HCC equally in open hepatectomy, because oncological protocols should remain consistent. Therefore, mainly in Eastern countries, variable techniques for laparoscopic AH are undergoing rapid development $[28,29]$. Among them, the Glissonian pedicle transection method, which was proposed for open hepatectomy by Takasaki et al. [30], is very useful for laparoscopic AH.

The advantages of laparoscopic hepatectomy are not limited to cosmetic issues or to the reduction of pain resulting from the small incision. Unique laparoscopic caudodorsal view utilization and bleeding control by use of pneumoperitoneum pressure are two of the most significant advantages of laparoscopic hepatectomy. Because the origins of both the Glissonian tree and the hepatic veins are located at the back and extend toward the ventral side, we usually expose the main trunk first at the back. We then advance with parenchymal dissection toward the periphery by exposing the vessels continuously, while utilizing the magnified view from the caudodorsal side by a scope inserted through the navel trocar in laparoscopic hepatectomy [31]. Through this method, split injury of vessels is prevented, dissection proceeds following landmarks, and blood does not localize in the cutting plane. In addition, pneumoperitoneum pressure plays a role in controlling the bleeding from a hole in the hepatic vein that has been exposed on the cutting plane in $\mathrm{AH}$ [32]. Furthermore, even in partial hepatectomy, the exposure of major vessels around the tumor utilizing AH techniques is often better for deep-seated or invisible lesions because unexpected exposure of the tumor on the cutting plane can be avoided by creating a cutting plane on the side of exposed vessels[33].

In Eastern countries, hepatic surgeons who have obtained advanced skills in laparoscopic hepatectomy prefer performing AH through a laparoscopic approach in addition to more traditional techniques.

\section{Conclusion}

Recent advances in perioperative imaging techniques provide useful surgical information for liver resection. 3D-CT enables virtual surgery planning by presenting the image of the tumor and liver, and it enables the accurate estimation of regional liver volumes. ICGfluorescence imaging is used to visualize hepatic regions for resection and it enables the estimation of portal uptake in veno-occlusive regions. LLR has been increasingly gaining popularity for the treatment of HCC in clinical practice worldwide. Thus, the techniques and future technical innovations are expected to further improve the safety of liver resections.

\section{Conflict of Interest}

The authors disclose no conflicts. 


\section{Financial Support}

None.

\section{References}

1 Jemal A, Bray F, Center MM, Ferlay J, Ward E, Forman D: Global cancer statistics. CA Cancer J Clin 2011;61:6990.

2 Imamura H, Seyama Y, Kokudo N, Maema A, Sugawara Y, Sano K, Takayama T, Makuuchi M: One thousand fifty-six hepatectomies without mortality in 8 years. Arch Surg 2003;138:1198-1206, discussion 1206.

3 Wakabayashi G, Cherqui D, Geller DA, et al: Recommendations for laparoscopic liver resection: a report from the second international consensus conference held in Morioka. Ann Surg 2015;261:619-629.

4 Endo I, House MG, Klimstra DS, Gönen M, D’Angelica M, Dematteo RP, Fong Y, Blumgart LH, Jarnagin WR: Clinical significance of intraoperative bile duct margin assessment for hilar cholangiocarcinoma. Ann Surg Oncol 2008;15:2104-2112.

5 Kenjo A, Miyata H, Gotoh M, Kitagawa Y, Shimada M, Baba H, Tomita N, Kimura W, Sugihara K, Mori M: Risk stratification of 7,732 hepatectomy cases in 2011 from the National Clinical Database for Japan. J Am Coll Surg 2014;218:412-422.

6 Lang H, Radtke A, Hindennach M, Schroeder T, Frühauf NR, Malagó M, Bourquain H, Peitgen HO, Oldhafer KJ, Broelsch CE: Impact of virtual tumor resection and computer-assisted risk analysis on operation planning and intraoperative strategy in major hepatic resection. Arch Surg 2005;140:629-638, discussion 638.

7 Endo I, Shimada H, Sugita M, Fujii Y, Morioka D, Takeda K, Sugae S, Tanaka K, Togo S, Bourquain H, Peitgen HO: Role of three-dimensional imaging in operative planning for hilar cholangiocarcinoma. Surgery 2007;142:666-675.

8 Yoshioka Y, Ebata T, Yokoyama Y, Igami T, Sugawara G, Nagino M: “Supraportal” right posterior hepatic artery: an anatomic trap in hepatobiliary and transplant surgery. World J Surg 2011;35:1340-1344.

9 Verbeek FP, van der Vorst JR, Schaafsma BE, Hutteman M, Bonsing BA, van Leeuwen FW, Frangioni JV, van de Velde CJ, Swijnenburg RJ, Vahrmeijer AL: Image-guided hepatopancreatobiliary surgery using nearinfrared fluorescent light. J Hepatobiliary Pancreat Sci 2012;19:626-637.

10 Ishizawa T, Kokudo N: The beginning of a new era of digestive surgery guided by fluorescence imaging. Liver Cancer 2014;3:6-8.

11 Makuuchi M, Hasegawa H, Yamazaki S: Ultrasonically guided subsegmentectomy. Surg Gynecol Obstet 1985;161:346-350.

12 Sano K, Makuuchi M, Miki K, Maema A, Sugawara Y, Imamura H, Matsunami H, Takayama T: Evaluation of hepatic venous congestion: proposed indication criteria for hepatic vein reconstruction. Ann Surg 2002;236:241-247.

13 Miyata A, Ishizawa T, Tani K, Shimizu A, Kaneko J, Aoki T, Sakamoto Y, Sugawara Y, Hasegawa K, Kokudo N: Reappraisal of a Dye-Staining Technique for Anatomic Hepatectomy by the Concomitant Use of Indocyanine Green Fluorescence Imaging. J Am Coll Surg 2015;221:e27-e36.

14 Kawaguchi Y, Ishizawa T, Miyata Y, Yamashita S, Masuda K, Satou S, Tamura S, Kaneko J, Sakamoto Y, Aoki T, Hasegawa K, Sugawara Y, Kokudo N: Portal uptake function in veno-occlusive regions evaluated by realtime fluorescent imaging using indocyanine green. J Hepatol 2013;58:247-253.

15 Kawaguchi Y, Sugawara Y, Ishizawa T, Satou S, Kaneko J, Tamura S, Aoki T, Sakamoto Y, Hasegawa K, Kokudo $\mathrm{N}$ : Identification of veno-occlusive regions in a right liver graft after reconstruction of vein segments 5 and 8: application of indocyanine green fluorescence imaging. Liver Transpl 2013;19:778-779.

16 Bryant R, Laurent A, Tayar C, Cherqui D: Laparoscopic liver resection-understanding its role in current practice: the Henri Mondor Hospital experience. Ann Surg 2009;250:103-111.

17 Laurent A, Cherqui D, Lesurtel M, Brunetti F, Tayar C, Fagniez PL: Laparoscopic liver resection for subcapsular hepatocellular carcinoma complicating chronic liver disease. Arch Surg 2003;138:763-769, discussion 769.

18 Morise Z, Ciria R, Cherqui D, Chen KH, Belli G, Wakabayashi G: Can we expand the indications for laparoscopic liver resection? A systematic review and meta-analysis of laparoscopic liver resection for patients with hepatocellular carcinoma and chronic liver disease. J Hepatobiliary Pancreat Sci 2015;22:342-352.

19 Han HS, Shehta A, Ahn S, Yoon YS, Cho JY, Choi Y: Laparoscopic versus open liver resection for hepatocellular carcinoma: Case-matched study with propensity score matching. J Hepatol 2015;63:643-650.

20 Parks KR, Kuo YH, Davis JM, O’ Brien B, Hagopian EJ: Laparoscopic versus open liver resection: a metaanalysis of long-term outcome. HPB Oxf 2014;16:109-118.

21 Cherqui D, Laurent A, Mocellin N, Tayar C, Luciani A, Van Nhieu JT, Decaens T, Hurtova M, Memeo R, Mallat A, Duvoux C: Liver resection for transplantable hepatocellular carcinoma: long-term survival and role of secondary liver transplantation. Ann Surg 2009;250:738-746.

22 Soubrane O, Goumard C, Laurent A, Tranchart H, Truant S, Gayet B, Salloum C, Luc G, Dokmak S, Piardi T, Cherqui D, Dagher I, Boleslawski E, Vibert E, Sa Cunha A, Belghiti J, Pessaux P, Boelle PY, Scatton O: Laparoscopic resection of hepatocellular carcinoma: a French survey in 351 patients. HPB Oxf 2014;16:357-365. 
23 Forner A, Llovet JM, Bruix J: Hepatocellular carcinoma. Lancet 2012;379:1245-1255.

24 Del Gaudio M, Ercolani G, Ravaioli M, Cescon M, Lauro A, Vivarelli M, Zanello M, Cucchetti A, Vetrone G, Tuci F, Ramacciato G, Grazi GL, Pinna AD: Liver transplantation for recurrent hepatocellular carcinoma on cirrhosis after liver resection: University of Bologna experience. Am J Transplant 2008;8:1177-1185.

25 Fuks D, Dokmak S, Paradis V, Diouf M, Durand F, Belghiti J: Benefit of initial resection of hepatocellular carcinoma followed by transplantation in case of recurrence: an intention-to-treat analysis. Hepatology 2012;55:132-140.

26 Kluger MD, Salceda JA, Laurent A, Tayar C, Duvoux C, Decaens T, Luciani A, Van Nhieu JT, Azoulay D, Cherqui D: Liver resection for hepatocellular carcinoma in 313 Western patients: tumor biology and underlying liver rather than tumor size drive prognosis. J Hepatol 2015;62:1131-1140.

27 Laurent A, Tayar C, Andréoletti M, Lauzet JY, Merle JC, Cherqui D: Laparoscopic liver resection facilitates salvage liver transplantation for hepatocellular carcinoma. J Hepatobiliary Pancreat Surg 2009;16:310314.

28 Yoon YS, Han HS, Cho JY, Ahn KS: Total laparoscopic liver resection for hepatocellular carcinoma located in all segments of the liver. Surg Endosc 2010;24:1630-1637.

29 Honda G, Kurata M, Okuda Y, Kobayashi S, Tadano S, Yamaguchi T, Matsumoto H, Nakano D, Takahashi K: Totally laparoscopic hepatectomy exposing the major vessels. J Hepatobiliary Pancreat Sci 2013;20:435440.

30 Yamamoto M, Katagiri S, Ariizumi S, Kotera Y, Takahashi Y: Glissonean pedicle transection method for liver surgery (with video). J Hepatobiliary Pancreat Sci 2012;19:3-8.

31 Honda G, Kurata M, Okuda Y, Kobayashi S, Sakamoto K, Takahashi K: Totally laparoscopic anatomical hepatectomy exposing the major hepatic veins from the root side: a case of the right anterior sectorectomy (with video). J Gastrointest Surg 2014;18:1379-1380.

32 Kobayashi S, Honda G, Kurata M, Tadano S, Sakamoto K, Okuda Y, Abe K: An experimental study on the relationship among airway pressure, pneumoperitoneum pressure, and central venous pressure in pure laparoscopic hepatectomy. Ann Surg 2015; (in press).

33 Honda G, Kurata M, Okuda Y, Kobayashi S, Sakamoto K: Totally laparoscopic hepatectomy exposing the vessels around the tumor intended to secure the surgical margin. Surg Endosc 2014;28:1331-1332. 